\title{
ESTUDO DA EROSÃO NA MICROBACIA DO CEVEIRO (PIRACICABA, SP). II - INTERPRETAÇÃO DA TOLERÂNCIA DE PERDA DE SOLO UTILIZANDO O MÉTODO DO ÍNDICE DE TEMPO DE VIDA ${ }^{(1)}$
}

\author{
Mara de Andrade Marinho Weill ${ }^{(2)} \&$ Gerd Sparovek $^{(3)}$
}

\begin{abstract}
RESUMO
A erosão acelerada do solo, um processo basicamente induzido pela ação antrópica, muito contribui para a degradação da qualidade das terras aráveis em todo o mundo, além de constituir a principal fonte não pontual de poluição dos recursos hídricos superficiais. Considerando a demanda efetiva pelo desenvolvimento de indicadores para avaliação do impacto da erosão na qualidade do solo em sistemas de produção agrícola, este trabalho teve por objetivo desenvolver um índice, com valor prognóstico, para ser aplicado como uma ferramenta de planejamento na interpretação da tolerância de perda de solo em áreas agrícolas. Foi desenvolvido o método designado "Índice de Tempo de Vida do Solo", para se proceder ao diagnóstico da erosão em uma área predominantemente utilizada com a cultura da cana-de-açúcar no município de Piracicaba (SP). Na realização do trabalho, foram empregados geotécnicas e métodos de análise geoestatística, sendo o processamento e a análise dos dados efetivados em ambiente de sistema de informação geográfica do tipo matricial. As taxas anuais médias de perda de solo foram estimadas em trabalho anterior, empregando a equação universal de perda de solo (EUPS), com ajuste dos fatores do modelo às condições locais da área de estudo. Nos cálculos do índice de tempo de vida do solo, foi presumida uma taxa de renovação de $0,2 \mathrm{~mm} \mathrm{ano}^{-1}$ e foram analisadas duas profundidades, de 50 e de $100 \mathrm{~cm}$, consideradas mínimas para o uso agrícola. A avaliação da espessura do solum revelou que, na área de estudo, predominam solos pouco profundos, com médias ponderadas pelas áreas de
\end{abstract}

\footnotetext{
(1) Parte da Tese de Doutorado do primeiro autor, com bolsa do CNPq. Trabalho realizado com auxílio financeiro da Fundação de Amparo à Pesquisa do Estado de São Paulo - FAPESP. Trabalho apresentado na 3th International Conference on Land Degradation, ICLD3, Rio de Janeiro, 2003. Recebido para publicação em janeiro de 2006 e aprovado em novembro de 2007.

(2) Professora Doutora da Faculdade de Engenharia Agrícola da Universidade de Campinas - FEAGRI/UNICAMP. Cidade Universitária Zeferino Vaz, Caixa Postal 6011, CEP 13083-875 Campinas (SP). E-mail: mweill@agr.unicamp.br

(3) Professor Associado do Departamento de Solos e Nutrição de Plantas da Escola Superior de Agricultura "Luiz de Queiroz" ESALQ. Caixa Postal 09, Av. Pádua Dias 11, CEP 13418-900 Piracicaba (SP). E-mail: gerd@esalq.usp.br
} 
ocorrência de $78 \mathrm{~cm}$, solos ocupados com cana-de-açúcar, e de $72 \mathrm{~cm}$, solos ocupados com outros usos. A aplicação do índice de tempo de vida revelou que, adotando a profundidade crítica de $50 \mathrm{~cm}$, o tempo de vida médio do solo nas áreas ocupadas com cana-de-açúcar é de 178 anos, e que, mantida a expectativa atual de perdas, pouco mais de 70 anos serão suficientes para degradar o recurso em cerca de $50 \%$ da área cultivada com cana-de-açúcar (meia-vida do solo). Para a profundidade crítica de $100 \mathrm{~cm}$, a situação se agrava, e o tempo de vida médio do solo nas áreas ocupadas com cana-de-açúcar cai para apenas 102 anos e a meia-vida para 42 anos. A aplicação do método possibilitou ainda estimar em cerca de 19 e de $74 \%$ as proporções da área cultivada com cana-de-açúcar em que a atual situação já é de impacto permanente instalado (tempo de vida do solo zero), isto é, locais onde as taxas de perda de solo são superiores à taxa de renovação, e a espessura do solo já é inferior às profundidades críticas consideradas, no caso $50 \mathrm{~m} \mathrm{e} 100 \mathrm{~cm}$, respectivamente. Nas condições atuais de uso e manejo, a situação de conservação de recursos, em particular do solo, pôde ser caracterizada em apenas 7,6 ha ou em menos de 1 \% da área com cana-de-açúcar. A taxa de renovação do solo foi superior às taxas estimadas de perdas por erosão. Em mais de $99 \%$ da área ocupada com cana-de-açúcar, portanto, as taxas estimadas de perda de solo por erosão superam a taxa de renovação do solo $(p>r)$, caracterizando a degradação de recursos. $O$ índice proposto mostrou-se uma ferramenta promissora para interpretação da tolerância da perda de solo aplicada ao planejamento do uso agrícola em bases sustentáveis.

Termos de indexação: meia-vida do solo, EUPS, SIG, programa IDRISI.

\section{SUMMARY: EROSION STUDY INTHE CEVEIRO WATERSHED (PIRACICABA, SP). II - INTERPRETING SOIL LOSS TOLERANCE USING THE SOIL USEFUL LIFE INDEX METHODOLOGY}

Accelerated soil erosion, a process basically induced by human activities contributes greatly to the degradation of arable land quality throughout the world, and is the main nonpoint source of surface water resource pollution. Considering the effective demand for developing indicators to evaluate the impact of soil erosion on soil quality in agriculture production systems, the objective of this study was to develop an index with a predictive value to be applied as a planning tool for interpreting soil loss tolerance in agricultural areas. The "Soil Useful Life Index" was developed and applied to diagnose erosion in an area predominantly used for sugarcane cultivation in the Piracicaba county, São Paulo State, Brazil. Geotechnology and geostatistics analysis methods were used in this study and the data processed and analyzed in a geographic matrix information system. Average annual soil loss rates had been estimated in a previous study using the universal soil loss equation (USLE), adjusting the model factors to the local study conditions. A $0.2 \mathrm{~mm}$ year $\mathrm{r}^{-1}$ renewal rate was assumed in the soil useful life index calculation, and two depths considered minimal for agricultural purposes $(50$ and $100 \mathrm{~cm})$ were analyzed. The evaluation of soil thickness revealed that shallower soils are predominant in the study area, with average depths of $78 \mathrm{~cm}$ in areas occupied by sugarcane and $72 \mathrm{~cm}$ in areas used for other purposes. The application of the soil useful life index revealed that at the critical depth of $50 \mathrm{~cm}$, the useful life of soils occupied by sugarcane is 178 years. By maintaining the current soil loss expectations, little more than 70 years will be sufficient to degrade $50 \%$ of the area cultivated with sugarcane (soil half-life). The situation worsens at critical depths of $100 \mathrm{~cm}$. Average soil useful life in areas occupied by sugarcane falls to only 102 years, and soil half-life is only 42 years. The method also permitted an estimation of the proportions of areas with sugarcane where the impact is already permanent (zero soil useful life) at 19 and $74 \%$, that is, where soil loss rates exceed the soil renewal rate, and soil thickness is already less than the critical depths considered, 50 and $100 \mathrm{~cm}$, respectively. Furthermore, under the current use and management conditions, the resources conservation, that is the soil renewal rate is higher than the estimated soil loss rates by erosion, can be characterized in only 7.6 ha, or less than $1 \%$ of the area under sugarcane. Therefore, in more than $99 \%$ of the area occupied by sugarcane the estimated rates of soil loss by erosion exceed the soil renewal rate $(p>r)$, characterizing a situation of resource degradation. The proposed index 


\author{
proved to be a promising tool for interpreting soil loss tolerance in the planning of \\ sustainable agriculture.
}

Index terms: IDRISI software, GIS, soil half-life, USLE.

\section{INTRODUÇÃO}

Em áreas agrícolas, a erosão acelerada do solo é basicamente um processo induzido pelo manejo, e comumente inicia-se com a remoção da cobertura vegetal original para a implantação das culturas. $\mathrm{O}$ uso e manejo de terras produtivas em desacordo com a aptidão agrícola, e o uso intensivo de terras marginais são outros fatores agravantes do processo (Dregne, 1982; Graaf, 1996).

A perda de solo devida à erosão acelerada é um importante fator de degradação de terras agrícolas em todo o mundo, condicionando o declínio da qualidade do solo e de sua capacidade de desempenhar múltiplas funções (Bennet \& Loundermilk, 1938; El-Swaife \& Dangler, 1982; Lal, 1994; Lal, 1997). No ambiente externo à propriedade, a produção de sedimentos decorrente da erosão acelerada do solo representa a principal fonte não-pontual de poluição dos recursos hídricos superficiais (Clark II et al., 1985).

O solo é um recurso natural vital, disponível apenas em quantidade limitada, e não renovável em uma escala de tempo humana (Jenny, 1980; Shertz, 1983; Lal \& Stewart, 1992). Por tais razões, a degradação de sua qualidade pela erosão acelerada, sobretudo de sua qualidade física, representa impacto permanente na base do recurso.

O planejamento do uso das terras é essencial para a prevenção da instalação de impactos permanentes na qualidade das terras e do solo em particular. A realização de previsões possibilita a indicação de ações preventivas e, ou corretivas, atendendo a diferentes horizontes de planejamento.

Assim, em face da relevância do diagnóstico da erosão acelerada do solo e da necessidade de predição da instalação de impactos permanentes na base desse recurso, o método do índice de tempo de vida do solo (Sparovek et al., 1997; Weill, 1999) é apresentado como um critério operacional e objetivo, alternativo ao valor T (Wischmeier \& Smith, 1978; McComark et al., 1982) para interpretação da expectativa de perda de solo estimada pelos diferentes modelos de erosão (EUPS, RUSLE, WEPP, dentre outros).

Este trabalho teve por objetivos apresentar o método para a obtenção do índice de tempo de vida do solo e relatar os resultados de um estudo de caso realizado em uma área intensivamente cultivada com cana-deaçúcar na microbacia do Ceveiro, município de Piracicaba (SP), ilustrando a aplicação desse critério na interpretação e no diagnóstico da erosão.

\section{MATERIAL E MÉTODOS}

\section{Método do índice de tempo de vida do solo}

Este método é proposto como um critério operacional e objetivo para a avaliação da sustentabilidade de sistemas agrícolas, aplicável no diagnóstico e na avaliação do impacto da erosão acelerada do solo em dada área. Por suas características, o índice constitui uma ferramenta promissora para o planejamento do uso das terras em bases sustentáveis. Foi desenvolvido e aplicado em ambiente digital em uma plataforma de sistema de informação geográfica (SIG), utilizando o programa IDRISI, um SIG matricial, nas versões for Windows v.2.0 (Eastman, 1993) e 32 (Eastman, 1999).

Esse índice foi concebido a partir do conceito de tolerância de perda de solo descrita como uma função, a partir dos trabalhos de Stamey \& Smith (1964), Skidmore (1982) e Sparovek \& Jong van Lier (1997), e do conceito operacional de sustentabilidade, conforme apresentado por Hansen (1996) e Kruseman et al. (1996). O caráter operacional do referido índice na interpretação da tolerância de perda de solo e na avaliação da sustentabilidade de sistemas agrícolas deve-se ao fato de ser simultaneamente literal, orientado pelo sistema quantitativo e possuir caráter diagnóstico (cenário presente) e prognóstico (cenário futuro).

Trata-se de um índice calculado com base em taxas atuais de perda de solo estimadas por um modelo de erosão, em uma taxa média de renovação do solo e na espessura de solo que excede uma profundidade mínima pré-definida como crítica para finalidade agrícola. A condição crítica identifica a instalação de impacto permanente, no caso uma profundidade mínima abaixo da qual a qualidade do solo é severamente degradada, em prejuízo do desempenho de suas funções, no caso a produção agrícola.

O método proposto permite identificar e mapear três situações distintas: (1) locais onde a taxa de renovação do solo supera as taxas atuais de perda de solo estimadas pelo modelo de erosão, caracterizando a situação que se denominou de conservação dos recursos (uso adequado); (2) locais onde a espessura do solum (ou soma das espessuras dos horizontes $\mathrm{A}+\mathrm{B})$ no tempo atual já é inferior à profundidade crítica pré-definida, caracterizando a situação denominada tempo de vida do solo zero na condição atual; (3) locais onde as taxas atuais de perda de solo superam a taxa de sua renovação, caracterizando a 
situação que se denominou degradação dos recursos (indicativa de áreas para monitoramento e controle). O cálculo desse índice é efetuado nas áreas enquadradas na situação degradação de recursos, visando a estimar o tempo remanescente, em anos, para que a situação de impacto permanente previamente definida (profundidade mínima) seja atingida. A partir daí, o monitoramento e controle do processo nas áreas de degradação podem ser realizados de acordo com horizontes de planejamento definidos com base no tempo de vida do solo calculado.

\section{Seqüência metodológica para caracteriza- ção das situações de planejamento e cálcu- lo do índice de tempo de vida do solo}

Conversão das taxas atuais de perda de solo estimadas pelo modelo de erosão, no caso a EUPS (A, em $\mathrm{Mg} \mathrm{ha}^{-1}$ ano-1), em altura de solo (h, em $\mathrm{mm} \mathrm{ano}^{-1}$ ), de acordo com a expressão:

$$
\mathrm{h}=0,1^{*} \mathrm{~A}^{*} \mathrm{~d}^{-1}
$$

sendo $\mathrm{h}=$ perda de solo, em $\mathrm{mm} \mathrm{ano}^{-1} ; \mathrm{A}=$ taxa atual de perda de solo, em $\mathrm{Mg} \mathrm{ha}^{-1} \mathrm{ano}^{-1} ; \mathrm{d}=$ densidade do solo, em $\mathrm{g} \mathrm{cm}^{-3}$.

Obtenção da taxa "líquida" de perda de solo, pela expressão:

$$
\mathrm{h}_{\mathrm{l}}=\mathrm{h}-\mathrm{r}
$$

sendo $\mathrm{h}_{1}=$ taxa líquida de perda de solo, em $\mathrm{mm} \mathrm{ano}^{-1}$; $\mathrm{h}=$ perda de solo, em mm ano ${ }^{-1} ; \mathrm{r}=$ taxa de renovação do solo, em mm ano-1.

Obtenção das áreas em "situação de conservação de recursos", ou locais onde a taxa de renovação do solo $(r)$ supera a taxa atual de perda de solo (p), por meio de uma operação escalar de subtração no SIG entre as respectivas imagens.

Obtenção das áreas em "situação de degradação permanente instalada", definidas por tempo de vida do solo igual a zero, isto é, áreas em que a espessura do solo já é inferior à profundidade mínima definida como crítica, por meio de operação escalar de subtração no SIG entre a imagem "espessura do solo" e o valor da profundidade mínima definida como crítica.

Cálculo do tempo de vida do solo nas áreas em "situação de degradação" ou áreas indicadas para monitoramento e controle, por meio de operação de divisão no SIG entre as imagens "perda de solo" (em mm ano ${ }^{-1}$ ) e "espessuras líquidas de solo" (em mm, depois de descontado o valor da profundidade crítica pré-definida).

Aplicação do método do índice de tempo de vida do solo: estudo de caso em área da Microbacia do Ceveiro, Município de Piracicaba (SP)

A área de estudo corresponde à maior parte da microbacia do Córrego do Ceveiro, um afluente do Rio
Piracicaba, inserindo-se na região tradicionalmente produtora de cana-de-açúcar no município de Piracicaba (SP). Totalizando cerca de 1990,40 ha, o uso predominante das terras se dá com a cultura de cana-de-açúcar, que ocupa cerca de 1.319 ha ou $66 \%$ da área. O restante da área de estudo é ocupado com mata, vegetação ciliar, reflorestamento, pastagens, áreas urbanas e represas, de acordo com levantamento do uso das terras efetuado por Fiorio (1998).

O interesse em se proceder ao estudo de caso nesta área se justifica, em primeiro lugar, pelo fato de as condições locais do clima, dos solos e das práticas de manejo adotadas serem representativas de boa parte da situação encontrada na região produtora de canade-açúcar em Piracicaba. Outro fato é a constatação do adiantado estado de degradação do meio físico, conforme atesta sedimentação acentuada de um açude a jusante, que abastecia com água potável uma população local de cerca de 2.000 habitantes do Distrito de Artemis, e que precisou ser prematuramente desativado em virtude do assoreamento.

Para a aplicação do método e do cálculo do índice de tempo de vida do solo, foi considerada uma taxa média de renovação do solo de $0,2 \mathrm{~mm}$ ano-1 (Skidmore, 1982). As taxas atuais de perda de solo foram estimadas pela EUPS, conforme descrito em trabalho anterior de Weill \& Sparovek (2008). O mapa da profundidade do solum (ou soma das espessuras dos horizontes A + B) foi obtido por interpolação pelo método de krigeagem ordinária, com base nos valores caracterizados por prospecção em 251 pontos na área de estudo. O cálculo das semivariâncias e a interpolação dos dados foram realizados utilizando o programa desenvolvido por Vieira ${ }^{(4)}$. O ajuste do semivariograma foi efetuado em planilha eletrônica (Excel ${ }^{\circledR}$ versão 97, da Microsoft). Os dados interpolados foram exportados para o programa Surfer ${ }^{\circledR}$ (Surfer Mapping System win 32, 1993-95; Golden Software Inc.), gerando um arquivo do tipo .grd (asc II), que foi então importado no IDRISI. Para interpolação e construção dos arquivos no SIG, foi definido o tamanho do pixel, ou célula básica de armazenamento de dados, como de $20 \times 20 \mathrm{~m}$. O procedimento detalhado do tratamento geoestatístico dos dados consta do trabalho de Weill (1999). Para estudo de cenários e estabelecimento de horizontes de planejamento, foram definidas duas profundidades para análise, de 50 e de $100 \mathrm{~cm}$, consideradas críticas para a finalidade de uso agrícola. Esses valores foram estabelecidos tendo em vista a variação da profundidade média do solum caracterizada para a área de estudo e, ainda, tomando por base os resultados obtidos por Salviano et al. (1998), que trabalharam em áreas muito erodidas no município de Piracicaba, utilizadas com cana-deaçúcar por cerca de 50 anos. Esses autores constataram

(4) Programa disponibilizado pelo Dr. Sidney Rosa Vieira, pesquisador do Instituto Agronômico (SP). 
que a produção de Crotalaria juncea L. foi reduzida a $65 \%$ do valor máximo nos locais submetidos à erosão severa, com exposição do horizonte C; que a produtividade não foi afetada onde os solos apresentavam $60 \mathrm{~cm}$ ou mais de espessura do solum; e que uma forte correlação com produtividades baixas ou muito baixas somente ocorreu nos solos com menos de $20 \mathrm{~cm}$ de espessura do solum.

No SIG, fez-se a separação das áreas com cana-deaçúcar das áreas com outros cultivos, situações que foram analisadas separadamente, e reunidas ao final para compor os mapas das situações de planejamento e de tempo de vida do solo na área de estudo, para as duas profundidades analisadas. A partir dos mapas de tempo de vida do solo, foram calculados no SIG histogramas de freqüência, discriminando as áreas de ocorrência por intervalo de tempo de vida do solo. $\mathrm{O}$ conjunto de informações foi tratado em planilha eletrônica, sendo obtidos gráficos demonstrativos integrando toda a informação gerada para avaliação do impacto da erosão acelerada na área de estudo.

\section{RESULTADOS E DISCUSSÃO}

\section{Caracterização das situações de planejamen- to na área de estudo}

A figura 1 apresenta o semivariograma ajustado aos dados de espessura do solum, e a figura 2, o mapa da variação da espessura do solum, na área de estudo, interpolado por krigeagem ordinária a partir dos parâmetros do semivariograma. Nas áreas ocupadas com cana-de-açúcar, a profundidade média dos solos, ponderada pela área de ocorrência, é de apenas $78 \mathrm{~cm}$, com valores mínimo de $26 \mathrm{~cm}$ e máximo de $184 \mathrm{~cm} \mathrm{e}$ elevado coeficiente de variação $(\mathrm{CV}=36,97 \%)$. Nas áreas com outros usos, a profundidade média do solum é ainda menor, de $72 \mathrm{~cm}$, variando entre o mínimo de

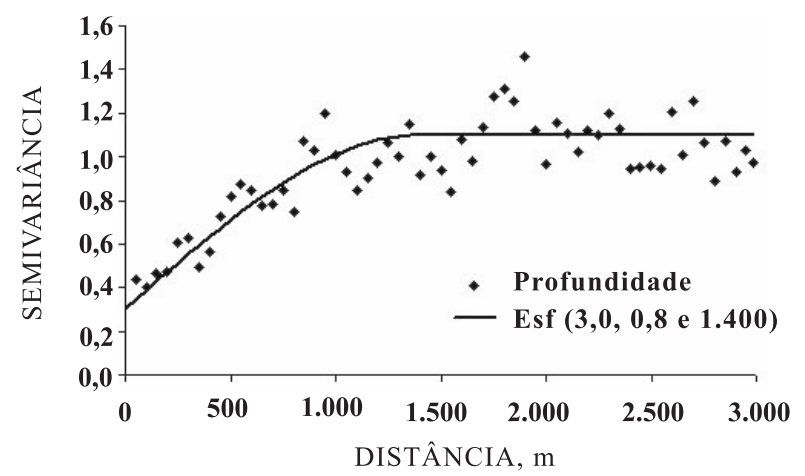

Figura 1. Semivariograma ajustado aos dados de profundidade do solum $(\mathrm{A}+\mathrm{B})$ para a área de estudo (Piracicaba, SP). (Modelo esférico; parâmetros $\left.C_{0}=0,3 ; C_{1}=0,8 ; a=1400\right)$.
$23 \mathrm{~cm}$ e o máximo de $172 \mathrm{~cm}(\mathrm{CV}=39,40 \%)$. Os solos mais espessos, com profundidade superior a $90 \mathrm{~cm}$ (Figura 2), correspondem aos Argissolos Vermelho-Amarelos e a uma pequena parte de Nitossolos Vermelhos na área, enquanto os solos rasos, com profundidade de até $50 \mathrm{~cm}$, são representados pelos Neossolos Litólicos; aqueles com profundidade entre 50 e $100 \mathrm{~cm}$ correspondem a Cambissolos Háplicos identificados na área.

O mapa das situações de planejamento da área de estudo é apresentado na figura 3. Nas áreas ocupadas com cana-de-açúcar, foi caracterizada situação de conservação em apenas 7,6 ha $(0,6 \%$ da área com cana), nos quais a taxa de renovação do solo é superior às taxas estimadas de perdas de solo por erosão $(r>p)$, considerando-se que os solos estão se espessando. Em mais de 99 \% da área ocupada com cana-de-açúcar, portanto, as taxas estimadas de perda de solo por erosão superam a taxa de renovação do solo $(p>r)$ caracterizando situação de degradação de recursos em particular do solo.

Para a profundidade crítica de $50 \mathrm{~cm}$, os solos já são mais rasos em cerca de 256 ha, caracterizando impacto permanente ou tempo de vida do solo zero em 19,5 \% da área com cana-de-açúcar. A área de controle e monitoramento para cálculo do tempo de vida do solo neste cenário totaliza 1.056,24 ha (80,50\%), sendo p > r; os solos possuem mais de $50 \mathrm{~cm}$ de espessura do solum.

A análise para a profundidade crítica de $100 \mathrm{~cm}$ mostrou que dos 1.312,04 ha com cana-de-açúcar, com p > r, a espessura do solum é inferior a $100 \mathrm{~cm} \mathrm{em}$ 973,84 ha, definindo situação de impacto permanente ou tempo de vida do solo zero em 73,8 \% da área cultivada com cana-de-açúcar. Aárea de controle para cálculo do tempo de vida do solo neste cenário se restringe somente a cerca de 338,20 ha $(25,78 \%)$, sendo p > r; o solum é mais espesso do que $100 \mathrm{~cm}$.

Nas áreas com outros usos, que ocupam 626,08 ha da área de estudo, a taxa de renovação do solo é superior às taxas estimadas de perda de solo por erosão $(r>p)$ em cerca de 499 ha, o que caracteriza situação de conservação de recursos em 79,71 \% das áreas ocupadas com mata, vegetação ciliar, reflorestamento e pastagens. Para os 127,04 ha restantes $(20,29 \%)$ da área sem cana-de-açúcar, foi caracterizada situação de degradação de recursos, em particular do solo, e as taxas estimadas de erosão superam a taxa de renovação do solo $(p>r)$.

Nas áreas com outros usos, a análise para a profundidade crítica de $50 \mathrm{~cm}$ indica que a área de controle para cálculo do tempo de vida do solo totaliza apenas 100 ha, onde os solos são mais profundos do que $50 \mathrm{~cm}$ e p > r. Em 27,08 ha, a ocorrência de solos mais rasos indica situação de impacto permanente ou tempo de vida do solo zero. Para a profundidade crítica de $100 \mathrm{~cm}$, a área de controle se reduz a 5,24 ha (4,12\%), onde o solumé mais profundo do que $100 \mathrm{~cm}$, indicando 


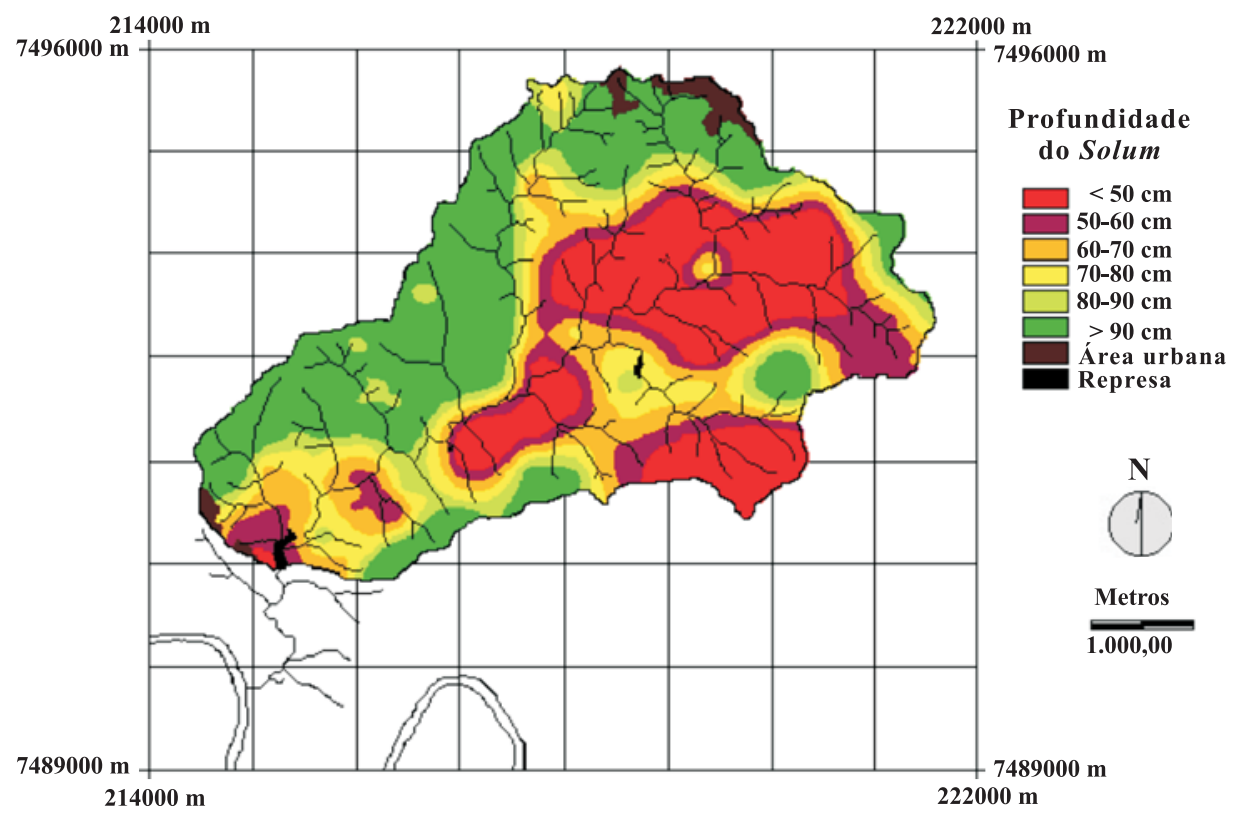

Figura 2. Mapa da profundidade do solum (soma das espessuras dos horizontes A + B) na área de estudo.

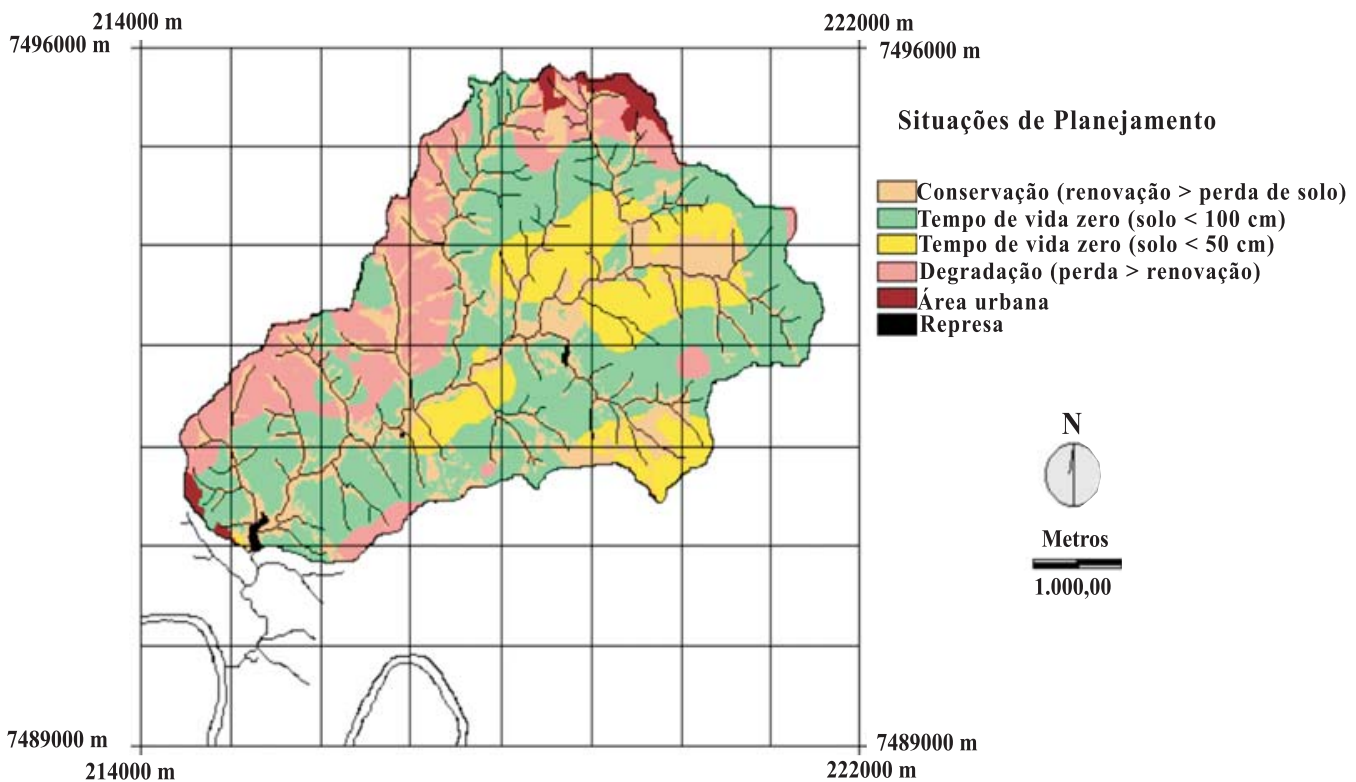

Figura 3. Mapa das situações de planejamento da área de estudo, de acordo com o método do índice de tempo de vida do solo.

que em mais de $95 \%$ da área com outros usos, que totalizam 121,80 ha, a situação é de tempo de vida do solo zero atualmente na profundidade considerada.

Para a área de estudo como um todo, foi caracterizada degradação de recursos nas condições de uso e manejo atuais, em 1.439,08 ha, onde as perdas por erosão superam a renovação do solo $(p>r)$. Considerando a profundidade crítica de $50 \mathrm{~cm}$, a área de controle para cálculo do tempo de vida do solo é de $1.156,20$ ha. Neste cenário, os restantes 282,88 ha correspondem a solos mais rasos, o que define situação de impacto permanente ou tempo de vida do solo zero. Para a profundidade crítica de $100 \mathrm{~cm}$, a área de controle é de apenas 343,54 ha, sendo a situação tempo de vida do solo zero caracterizada em 1.095,54 ha (Quadro 1).

Os resultados obtidos indicam que a cultura da cana-de-açúcar representa o uso que exerce a maior pressão sobre a qualidade do solo na área de estudo, uma vez que, em 99,4\% da área utilizada com a cultura, as taxas de perda de solo superam a taxa de sua renovação. Os solos estão ficando mais rasos às 
Quadro 1. Comparação entre as taxas estimadas de perda de solo (p) e a taxa de renovação do solo (r) em termos da área de ocorrência, para as áreas com cana-de-açúcar e com outros usos para a área de estudo

\begin{tabular}{|c|c|c|c|c|c|}
\hline \multirow{4}{*}{ Local } & \multicolumn{5}{|c|}{ Área } \\
\hline & \multirow{3}{*}{ Total } & \multirow{3}{*}{$\begin{array}{c}\text { Conservação de recursos } \\
\qquad \mathbf{r}>\mathbf{p}^{(1)}\end{array}$} & \multicolumn{3}{|c|}{ Degradação de recursos $(p>r)$} \\
\hline & & & \multirow[t]{2}{*}{ Total } & \multicolumn{2}{|c|}{ Solum } \\
\hline & & & & $<50 \mathrm{~cm}$ & $<100 \mathrm{~cm}$ \\
\hline Cana-de-açúcar & $1.319,63$ & 7,6 & $1.312,04$ & 255,80 & 973,84 \\
\hline Outros usos & 626,08 & 499,04 & 127,04 & 27,08 & 121,80 \\
\hline Total na área & $1.945,72$ & 506,64 & $1.439,08$ & 282,88 & $1.095,64$ \\
\hline
\end{tabular}

custas da perda do horizonte superficial, em geral o mais fértil e rico em matéria orgânica. O fato de que predominantemente os solos da área de estudo já são rasos, ou pouco profundos, evidencia a importância do controle da erosão no sentido da redução da expectativa atual de perda de solo para níveis mais toleráveis. Para uso agrícola sustentável, e considerando a profundidade do solo como um atributo não renovável em um tempo compatível com o da existência humana, as áreas enquadradas na situação de impacto permanente, ou tempo de vida do solo zero, são indicadas para usos menos intensivos, em especial onde a espessura do solum já é inferior a $50 \mathrm{~cm}$.

\section{Tempo de vida do solo na área de estudo}

O índice de tempo de vida do solo remete ao tempo para atingir a profundidade definida como crítica ao uso agrícola. A figura 4 ilustra a espacialização do tempo de vida do solo considerando a profundidade crítica de $50 \mathrm{~cm}$. Neste cenário, nas áreas de controle e monitoramento ocupadas com a cultura da cana-deaçúcar, o tempo de vida médio do solo, ponderado pela área de ocorrência, foi calculado como de 178 anos. Os resultados obtidos revelam ainda que, mantidas as condições atuais de uso e manejo, pouco mais de 70 anos serão suficientes para degradar o solo em cerca de $50 \%$ da área com cana-de-açúcar (meia-vida do solo). Considerando que $50 \mathrm{~cm}$ já é uma espessura restrita, a aplicação do método indica que em até três gerações metade da área de estudo hoje ocupada com a cultura estará inviabilizada para uso agrícola. Além disso, pode-se presumir que boa parte do sedimento gerado na área agrícola se transformará em fonte não pontual de poluição, acarretando a degradação da qualidade da água no ambiente externo e diminuição de sua disponibilidade para usos diversos.
Adotando $100 \mathrm{~cm}$ como profundidade crítica, o tempo de vida do solo médio na área de controle e monitoramento, no total de 338,20 ha, foi calculado como de apenas 102 anos. Neste novo cenário, mantida a expectativa atual de perda de solo, a meiavida do solo ou tempo estimado para que ocorra a degradação de cerca de metade da área avaliada se reduz para 42 anos. O mapa do tempo de vida do solo para a profundidade crítica de $100 \mathrm{~cm}$ é apresentado na figura 5.

Tendo em vista que a taxa anual média de perda de solo, ponderada pela área de ocorrência, é de cerca de $54 \mathrm{Mg} \mathrm{ha}^{-1}$ ano $^{-1}$ nas áreas de controle e monitoramento, tanto para a profundidade crítica de $50 \mathrm{~cm}$ quanto para a de $100 \mathrm{~cm}$, a redução do tempo de vida médio para $43 \%$ e da meia-vida do solo para $40 \%$ nas profundidades com mais de $100 \mathrm{~cm}$ ocorre porque as espessuras são pouco superiores à profundidade considerada como crítica $(100 \mathrm{~cm})$, e o esperado é que, em pouco tempo, o "excedente" de espessura do solo poderá ser perdido por erosão.

Para as áreas com outros usos, com pastagens, reflorestamento, vegetação ciliar e mata, correspondentes a cerca de 100 ha, considerando a profundidade crítica de $50 \mathrm{~cm}$, o tempo de vida do solo médio, ponderado pela área, é superior a 16.000 anos, e para a metade da área (50 ha), o tempo de vida do solo (meiavida do solo) é pouco inferior a 6.000 anos. Considerando a profundidade de $100 \mathrm{~cm}$, o tempo de vida do solo médio, ponderado pela área de ocorrência, passa a ser de 29.000 anos, referindo-se, entretanto, a uma área avaliada de apenas 5,24 ha (em que $\mathrm{p}>\mathrm{r}$ e a profundidade do solum é maior do que $100 \mathrm{~cm}$ ) (menos de $0,3 \%$ da área de estudo). Os resultados para a área de controle e monitoramento da perda de solo com outros usos indicam que as taxas de perda de solo são apenas pouco superiores à taxa de renovação 


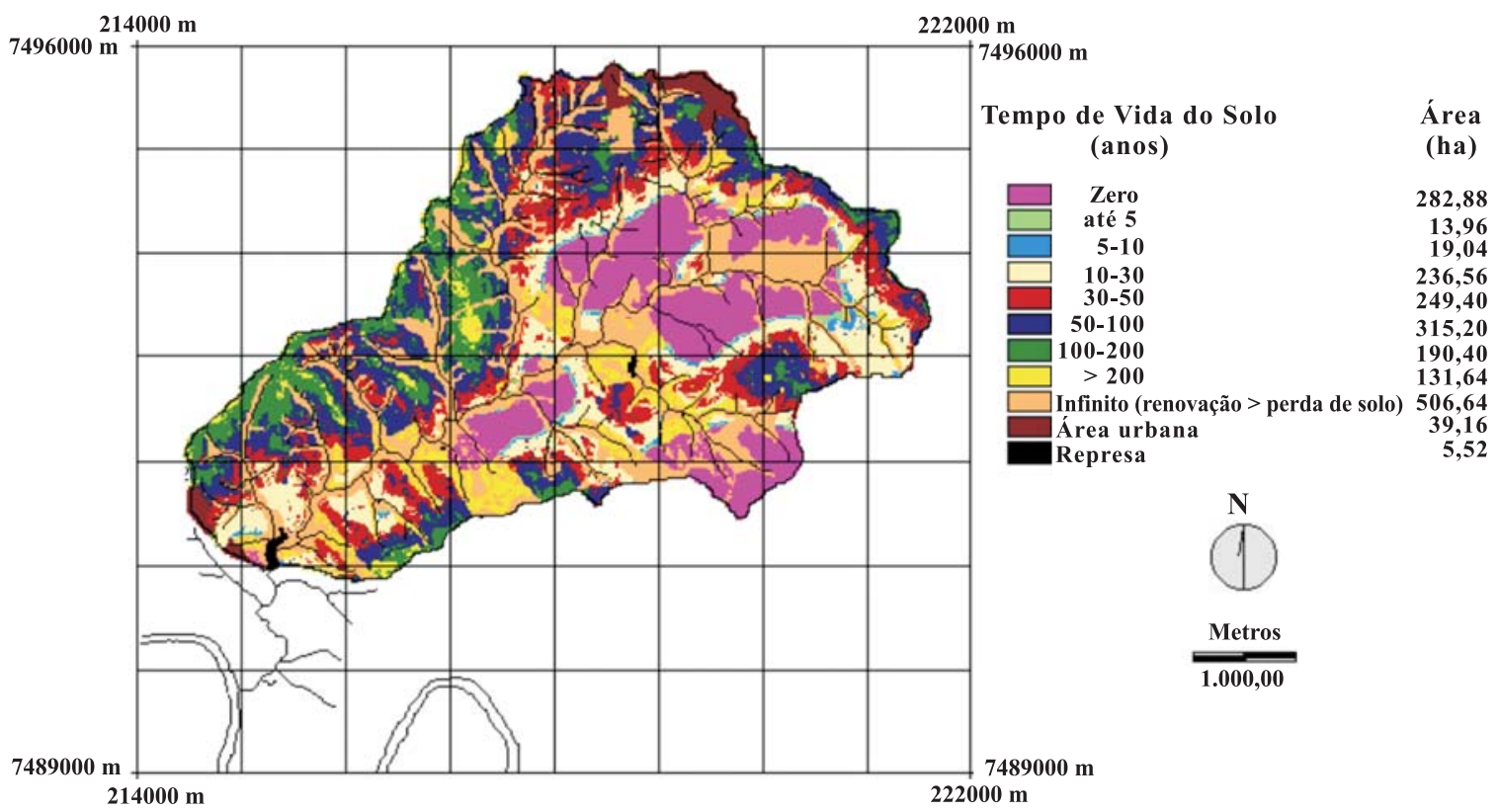

Figura 4. Mapa do tempo de vida do solo (em ano) da área de estudo na profundidade crítica de $50 \mathrm{~cm}$.

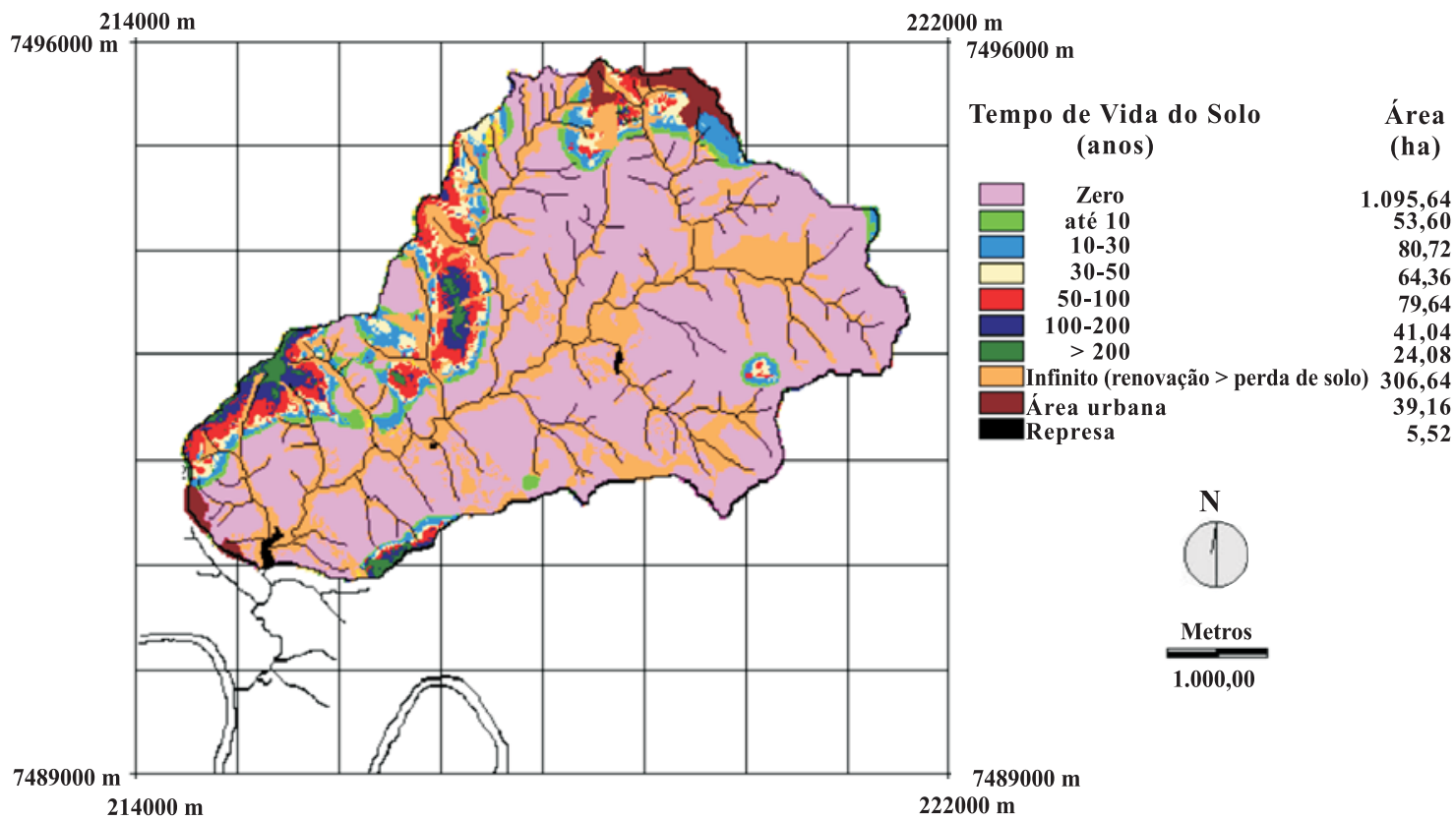

Figura 5. Mapa do tempo de vida do solo (em ano) da área de estudo para a profundidade crítica de $100 \mathrm{~cm}$.

do solo, uma vez que milhares de anos serão necessários para que ocorra a perda do solo excedente.

Para a área de estudo como um todo, a situação é intermediária aos dois extremos, em que há solos degradando-se rapidamente (nas áreas com cana-de-açúcar) e outros muito lentamente (nas áreas com outros usos). O tempo de vida do solo médio, ponderado pela área, é de 1.600 anos para a profundidade crítica de
$50 \mathrm{~cm}$ (cenário 1), mas a metade da área avaliada (578,10 ha) já deverá estar severamente degradada em até 140 anos (meia-vida do solo). A análise para a profundidade crítica de $100 \mathrm{~cm}$ (cenário 2) revelou que o tempo de vida do solo médio ponderado é de 551 anos, e 70 \% da área (240,41 ha) deverá ser severamente degradada em até 81 anos. Eliminando os cinco valores mais altos de tempo de vida do solo, superiores a 65.000 anos, mas que representam somente 0,2 ha da 
área de estudo, o tempo de vida do solo médio ponderado cai para 296 anos e a meia-vida do solo é inferior a 43 anos.

As figuras 6 e 7 ilustram os gráficos do tempo de vida do solo (em anos) considerando, respectivamente, as profundidades críticas de 50 e de $100 \mathrm{~cm}$. Na interpretação do gráfico, na ordenada onde $\mathrm{x}=0$ (tempo de vida do solo zero), o valor de $\mathrm{AR}$ (área relativa) indica a proporção de área degradada até a profundidade crítica de hoje. No patamar do gráfico, onde a curva se estabiliza, o valor da área relativa indica a máxima proporção da área passível de ser degradada por erosão até a situação extrema considerada. Em outras palavras, se a curva estabiliza ao redor de 0,5, significa que no máximo 50 \% da área avaliada poderá ser degradada por erosão ao extremo, e para a outra metade, a condição atual é de conservação de recursos, no caso do solo. A inclinação da curva indica a intensidade da erosão, e o cálculo da tangente fornece a taxa de perda de solo no ponto considerado.

O gráfico da figura 6 demonstra, para a quase totalidade das áreas com outros usos, a situação é de conservação dos recursos, e, na pequena porção da área onde as taxas de perda de solo estimadas superam a taxa de renovação $(p>r)$, a intensidade do processo é muito baixa, uma vez que decorrerão milhares de anos sem que ocorra alteração significativa da área relativa. Também é evidente que a proporção da área que configura impacto irreversível atualmente é muito pequena, inferior a 0,05. Para as áreas utilizadas com cana-de-açúcar, apenas uma ínfima porção se encontra em situação de conservação de recursos $(<0,01)$, significando que, para mais de $99 \%$ da área, a degradação até a situação extrema é uma possibilidade concreta. Caracterizou-se, tempo de vida do solo zero em 19 \% da área (ponto de interseção da curva com o eixo y), onde a espessura do solum já é inferior a $50 \mathrm{~cm}$. Entre os dois extremos, a inclinação

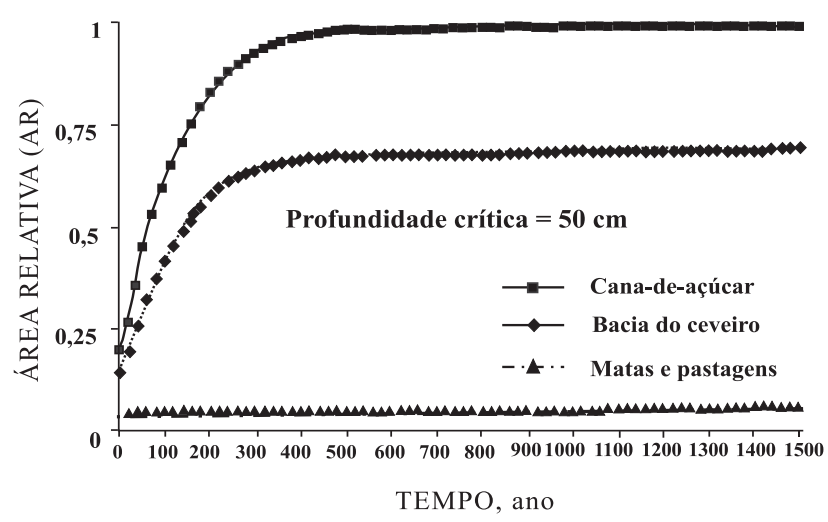

Figura 6. Gráfico do tempo de vida em anos para as áreas com cana-de-açúcar, com outros usos e para a área de estudo como um todo, considerando a profundidade crítica de $50 \mathrm{~cm}$.

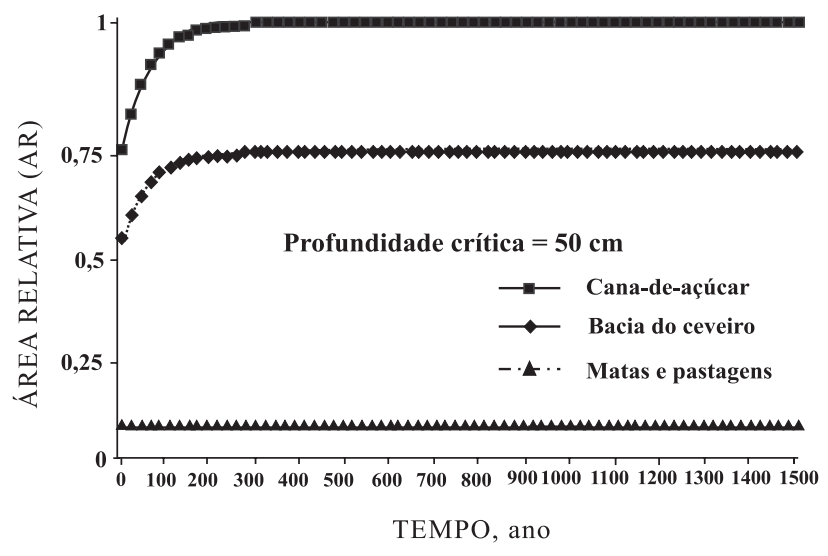

Figura 7. Gráfico do tempo de vida em anos nas áreas com cana-de-açúcar, com outros usos e na área de estudo como um todo, considerando a profundidade crítica de $100 \mathrm{~cm}$.

da curva do tempo de vida do solo indica processo intenso de degradação, que pode condicionar impacto permanente em quase $80 \%$ da área com cana-deaçúcar em menos de 200 anos. Para a área de estudo como um todo, está indicado tempo de vida do solo zero em $14,54 \%$ da área e patamar ao redor de $\mathrm{AR}=$ 0,74 , indicando que cerca de $26 \%$ da área avaliada conserva o solo. A inclinação da curva indica processo acelerado de degradação, mas menos intenso comparativamente às áreas com cana-de-açúcar, com projeção de que 50 \% da área deverá ser degradada ao extremo num intervalo de apenas 140 anos.

A figura 7 resume os resultados obtidos considerando o cenário em que a profundidade crítica é de $100 \mathrm{~cm}$. Nas áreas com outros usos (mata, vegetação ciliar, reflorestamento e pastagens), há registro de tempo de vida do solo zero em cerca de $20 \%$ da área $(\mathrm{AR}=0,1945)$. Nas áreas com cana-de-açúcar, foi caracterizado tempo de vida do solo zero em $74 \%$ da área $(\mathrm{AR}=0,7380)$ e, para a área de estudo como um todo, esse tempo foi caracterizado em $56 \%$ da área $(\mathrm{AR}=0,5631)$.

O diagnóstico da erosão acelerada na área de estudo, aplicando o método do índice de tempo de vida do solo, contém forte indicativo de que o estado de degradação das áreas ocupadas com a cultura de cana-de-açúcar é elevado e de que o processo instalado de erosão é muito intenso, sendo recomendável uma intervenção na área, com vistas à preservação do que ainda resta da base do recurso solo. A qualidade do solo e, por conseguinte, a do ambiente circundante encontramse em processo de declínio e, a julgar pelos dados, a sustentabilidade do uso agrícola na área está ameaçada. A implementação de um plano de controle da erosão e de recuperação, baseado na revisão do uso e das práticas de manejo agrícola adotados, poderá evitar que seja atingida a degradação extrema, conforme caracterizado para a maior parte da área analisada. 
A aplicação do método do índice de tempo de vida na área de estudo trouxe subsídios para o diagnóstico da erosão instalada, permitindo identificar o uso mais impactante; as áreas com indicação imediata para intervenção e controle da erosão; e, ainda, a proporção da área e o tempo remanescente para que a degradação extrema considerada seja atingida, caso medidas corretivas não sejam implementadas.

\section{CONCLUSÕES}

1. O método do índice de tempo de vida do solo mostrou-se um critério alternativo ao valor $\mathrm{T}$, para interpretação da tolerância das taxas estimadas de perda de solo pelos modelos de erosão, no caso a EUPS.

2. O método do índice de tempo de vida do solo também mostrou-se adequado e operacional para aplicação no planejamento sustentável do uso agrícola da terra, por permitir horizontes de planejamento, com valor diagnóstico, para avaliação de cenários atuais, e prognóstico, para avaliação de cenários futuros.

\section{AGRADECIMENTOS}

Ao Dr. Sidney Rosa Vieira, pesquisador do Centro de Solos e Recursos Agroambientais do Instituto Agronômico, pela colaboração no tratamento geoestatístico dos dados.

\section{LITERATURA CITADA}

BENNET, H.H. \& LOUDERMILK, W.C. General aspects of the soil-erosion problem. In: Soils and men. Washington, USDA, Yearbook of Agriculture, 1938. p.581-608.

CLARK II, E.H.; HAVERKAMP, J.A. \& CHAPMAN, W. Eroding soils. The off-farm impacts. Washington, The Conservation Foundation, 1985. 252p.

DREGNE, H.E. Historical perspective of accelerated erosion and effect on world civilization. ASAE Spec. Public., 45:114, 1982.

EASTMAN, J.R. IDRISI 32 - Guide to GIS and image processing. Worcester, Clark University, 1999. 193p.

EASTMAN, J.R. IDRISI manual. Massachusetts, Graduate School of Geography, Clark University, 1993.

EL-SWAIFE, S.A. \& DANGLER, E.W. Rainfall erosion in the tropics: A state-of-the-art. In: KUSSOW, W.; EL-SWAIFY, S.A. \& MANNERING, J., eds. Soil erosion and conservation in the tropics. Madison, American Society of Agronomy, 1982. p.1-25.

FIORIO, P.R. Avaliação cronológica do uso da terra e seu impacto no ambiente da microbacia hidrográfica do Ceveiro da região de Piracicaba, SP. Piracicaba, Escola Superior de Agricultura Luiz de Queiroz, 1998. 114p. (Tese de Mestrado)
GRAAF, J. The price of soil erosion. An economic evaluation of soil conservation and watershed development. Netherlands Foundation for the Advancement of Tropical Research (WOTRO), Netherlands Organization for Scientific Research (NWO), Project number W 45-104, 1996. p.1-25.

HANSEN, J.W. Is agricultural sustainability a useful concept? Agric. Systems, 50:117-143, 1996.

JENNY, H. The soil resource: Origin and behavior. New York, Spring-Verlag, 1980. (Ecological Studies, 37)

KRUSEMAN, G.; RUBEN, R.; KUYVENHOVEN, A.; HENGSDIJK, H. \& van KEULEN, H. Analytical framework for disentangling the concept of sustainable land use. Agric. Systems, 50:191-207, 1996.

LAL, R. Soil erosion by wind and water: Problems and prospects. In: LAL, R., ed. Soil erosion research methods. 2.ed. Ankeny, Soil and Water Conservation Society, 1994. p.1-9.

LAL, R. Soil quality and sustainability. In: LAL, R.; BLUM, W.E.H.; VALENTIN, C. \& STEWART, B.A., eds. Methods of assessment of soil degradation. Boca Raton, CRC Press, 1997. p.17-30.

LAL, R. \& STEWART, B.A. Need for land restorarion. Adv. Soil Sci., 17:1-11, 1992.

McCORMACK, D.E.; YOUNG, K.K. \& KIMBERLIN, L.W. Current criteria for determining soil loss tolerance. Madison, American Society of Agricultural Engineers, 1982. (ASAE. Special Publication, 45)

SALVIANO, A.A.C.; VIEIRA, S.R. \& SPAROVEK, G. Variabilidade espacial de atributos de solo e de Crotalaria juncea L. em área severamente erodida. R. Bras. Ci. Solo, 22:115-122, 1998

SCHERTZ, D.L. The basis for soil loss tolerances. J. Soil Water Conser., 38:10-14, 1983.

SKIDMORE, E.L. Soil-loss tolerance. In: AMERICAN SOCIETY OF AGRONOMY. Determinants of soil-loss tolerance. Madison, 1982. p.87-93.

SPAROVEK, G. \& JONG VAN LIER, Q. Definition of tolerable soil erosion values. R. Bras. Ci. Solo, 21:467-471, 1997.

SPAROVEK, G.; WEILL, M.A.M.; RANIERI, S.B.L.; SCHNUG, E. \& SILVA, E.F. The time-life concept as a tool for erosion tolerance definiton. Sci. Agric., 54:130-135, 1997.

STAMEY, W.L. \& SMITH, R.M. A conservation definition of soil tolerance. Soil Sci., 97:183-186, 1964.

WEILL, M.A.M. Estimativa da erosão do solo e avaliação do seu impacto na microbacia do Ceveiro (Piracicaba, SP), através do Índice de Tempo de Vida. Piracicaba, Escola Superior de Agricultura "Luiz de Queiroz", 1999. 100 p. (Tese de Doutorado)

WEILL, M.A.M. \& SPAROVEK, G. Estudo da erosão na Microbacia do Ceveiro (Piracicaba, SP). I- Estimativa das taxas de perda de solo e estudo de sensibilidade dos fatores do modelo EUPS. R. Bras. Ci. Solo, 32:801-814, 2008.

WISCHMEIER, W.H. \& SMITH, D.D. Predicting rainfall erosion losses - A guide to conservation planning. Washington, Department of Agriculture, 1978. (Agricultural Handbook, 537) 